Goldschmidt 2021 Abstract

https://doi.org/10.7185/gold2021.8181

\section{Assessing soil organic matter features under drought and normal conditions using direct infusion high resolution mass spectrometry and LC-MS/MS feature based molecular networking}

\author{
NICOLE DIDONATO $^{1}$, ALBERT RIVAS UBACH ${ }^{1}$, \\ CHAEVIEN CLENDINEN ${ }^{1}$, NOAH SOKOL ${ }^{2}$, NIKOLA

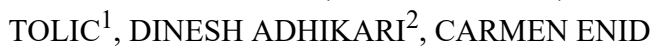 \\ MARTINEZ $^{3}$, JENNIFER PETT-RIDGE ${ }^{2}$ AND LJILJANA \\ PASA TOLIC ${ }^{1}$ \\ ${ }^{1}$ Pacific Northwest National Laboratory \\ ${ }^{2}$ Lawrence Livermore National Laboratory \\ ${ }^{3}$ Cornell University \\ Presenting Author: nicole.didonato@pnnl.gov
}

Knowledge of the type of carbon that escapes mineralization and persists in soils is important for predicting carbon fluxes in a warming climate. Direct infusion FTICRMS enables observations of broad differences in compositions of soil organic matter molecular formulas among treatments ${ }^{1}$. Although complete separation of soil organic matter is not yet achievable, LC-MS/MS compliments direct infusion by reducing charge competition, improving feature detection and allowing for molecular identification ${ }^{2}$. However, only a fraction of soil organic matter features can be identified due to the lack of comprehensive libraries ${ }^{3}$. Molecular networking can further improve identifcation of unknown compounds by matching their fragmentation spectra with similar spectra of known compounds.

In this work, LC-MS/MS feature based molecular networking ${ }^{4}$ is used to investigate soil organic matter features from plant-soil microcosms subject to drought and normal conditions. Networked features are compared with direct infusion features to corroborate molecular formula assignments and provide additional structural information of unknowns. The fraction of formulas observed by direct infusion that can be separated, matched with existing libraries or categorized by neutral losses is also assessed.

Preliminary results suggest a significant portion of direct infusion features can be separated using chromatography and 5$30 \%$ of direct infusion assigned formulas per sample could be networked using global natural product social (GNPS) feature based molecular networking. We found good agreement between GNPS annotations and direct infusion molecular formula assignments. Unique annotations include polyphenols, lipids, terpenoids and carboxylic acids, expected components of natural organic matter. Although multiple orthogonal chromatographic separations are required for a comprehensive evaluation of the diversity of features, LC-MS-MS molecular networking validates direct infusion formula assignments, improves assignment rates and enables structural information to be inferred. This analysis, combined with the potential for expanding libraries of known components of soil organic matter are the next steps to understanding the molecular structures of carbon that persist in soils.
[1] Tfaily, M. M., et al. (2013). Geochim et Cosmochim Acta 112: $116-129$.

[2] Lu, K. and Z. Liu (2019). Front Mar Sci 6(673).

[3] Nguyen, T. D., et al. (2020). Metabolites 10(3): 86.

[4] Wang, $M$ et al. (2016) Nat Biotechnol 34.8: 828-837 